\title{
Hibridación de los modelos de Educación Deportivay Responsabilidad Personal y Social: una experiencia a través de un programa de kickboxing educativo Hybridizing Sport Education and Teaching for Personal and Social Responsibility: an experience through an educational kickboxing program \\ José Ignacio Menéndez Santurio, Javier Fernández-Río \\ Universidad de Oviedo (España)
}

\begin{abstract}
Resumen. La Educación Física actual demanda planteamientos innovadores, tanto desde el punto de vista de la metodología como del contenido, que enganchen a los estudiantes y hagan suyo el proceso de enseñanza-aprendizaje. El presente artículo presenta una experiencia práctica de hibridación de dos modelos pedagógicos, Educación Deportiva y Responsabilidad Personal y Social, a través de un contenido novedoso: el kickboxing educativo (sin contacto). Esta se desarrolló a través de una unidad didáctica de 16 sesiones aplicada a cinco grupos naturales de $4^{\circ}$ de la ESO (15-17 años). Se describen profundamente todos los elementos fundamentales de la experiencia llevada a cabo para que pueda ser analizada, valorada y desarrollada en otros contextos de Educación Física. Lo observado durante la experimentación permite afirmar que la combinación o hibridación de los modelos de Educación Deportiva y Responsabilidad Personal y Social posibilita transformar el aula en un contexto centrado en el estudiante. Así mismo, el kickboxing educativo (sin contacto) se ha mostrado como un contenido muy válido para la Educación Física.

Palabras clave. Educación Física, modelos pedagógicos, deportes de combate, artes marciales, innovación, Práctica Basada en Modelos.
\end{abstract}

Abstract. Contemporary Physical Education demands innovative approaches, both from the methodological and from the content point of view, to catch the attention of students and give them ownership over the teaching-learning process. This article presents a practical experience on the hybridization of two pedagogical models, Sport Education and Teaching for Personal and Social Responsibility, through a novel content: educational kickboxing (contactless). The practical experience was developed through a learning unit of 16 sessions applied to five intact groups in grade 10 (1517 years). The main elements of the experience are fully described so they can be analyzed, assessed and implemented in other physical education contexts. Observations of the experience allows us to say that the combination or hybridization of the Sport Education and Teaching for Personal and Social Responsibility models allows teachers to transform the class in a student-centered context. Educational kickboxing (contactless) has proven to be a very relevant content area for Physical Education.

Keywords. Physical Education, pedagogical models, combat sports, martial arts, innovation, Models-Based Practice.

\section{Introducción}

A lo largo del siglo XX, la enseñanza de la Educación Física(EF) ha estado basada principalmente en métodos de instrucción directa donde el protagonismo del alumnado era escaso. Estos métodos habían sido heredados de la instrucción militar y adaptados a los programas físicos (Van Dalen y Bannet, 1971). En los años setenta, importantes teóricos del área realizaron una renovación metodológica implantando nuevos conceptos como las estrategias y los estilos de enseñanza, que posibilitaron nuevas herramientas de trabajo para el docente en aras de escapar de la estructura tan hermética que otorgaba la instrucción directa (Mosston y Ashworth, 2002). Pasaron por tanto de existir formas de enseñanza basadas en el profesor a otras centradas en el alumnado. En los años 80, Joyce y Weil (1985, p.11) acuñaron la primera definición del término modelo, afirmando que se trataba de «un plan estructurado que puede usarse para configurar un currículum (curso de estudios a largo plazo), para diseñar materiales de enseñanza y para orientar la enseñanza en las aulas».

Posteriormente, autores del ámbito de la EF como Jewett y Metzler hablaron de los términos modelo curricular y modelo de instrucción como términos similares al modelo de enseñanza. Por una parte, Jetwett, Bain y Ennis (1995, p.15) afirmaban que un modelo curricular era:

Un patrón general para crear o dar forma a diseños de programas que se basa en un marco conceptual, incorporando la identificación de objetivos de aprendizaje y la selección y la estructuración del contenido del programa (...) incluye el desarrollo de procedimientos de enseñanza y ambientes de aprendizaje.

Por su parte, Metzler (2011, p.13) hablaba de modelo de instrucción refiriéndose a «una perspectiva de la intervención basada en teorías del aprendizaje, objetivos de aprendizaje a largo plazo, contexto de enseñanza, contenido, organización y control de clase, estrategias y estilos de enseñanza, evaluación del proceso y evaluación del aprendizaje del alumnado». Este tipo de planteamientos no apartan a los habi-

Fecha recepción: 17-09-15. Fecha de aceptación: 22-12-15 José Ignacio Menéndez Santurio UO194643@uniovi.es tualmente utilizados métodos, estilos y estrategias de enseñanza sino que los incorpora en un escenario más amplio que ofrecen los modelos (Fernández-Río, Méndez-Giménez, 2016). Actualmente este tipo de enfoques educativos son conocidos como Práctica basada en Modelos y poco a poco están siendo sustituidos por los enfoques tradicionales centrados en el docente (Hastie y Casey, 2014).

Por otra parte, este término puede ser utilizado en el deporte, en el ejercicio físico, en el contexto artístico o incluso en actividades de ocio extraescolares como por ejemplo, la danza o las actividades de aventura (Haerens, Kirk, Cardon y De Bourdeaudhuij, 2011). En la actualidad, autores como Haerens el al. (2011) prefieren hablar de modelos pedagógicos. Según ellos, el término instrucción procedente de la definición de Metzler (2005) mantiene el foco en el docente, mientras que el término curricular de Jetwett «mantiene el foco en el contenido y resta importancia al docente, especialmente porque los docentes suelen percibir el contenido como actividades de aprendizaje» (Jewett et al., 1995, p. 124). Utilizando el término pedagógico se destaca la interdependencia del aprendizaje, de la enseñanza, del contexto y del contenido (Rovegno, 2006).

El Modelo de Educación Deportiva (MED; Siedentop, 1994; Siedentop, Hastie y Van der Mars, 2004) es uno de los enfoques que más interés está recibiendo en los últimos años por parte de los investigadores en EF. Este modelo se centra en una serie de características que a pesar de estar muy presentes en el deporte institucionalizado, en la enseñanza deportiva escolar no se incluyen de forma sistemática. Estas son:(1) temporada: para favorecer el desarrollo de todos sus elementos, el MED trata, en primer lugar, de alargar las UDs, pasando de las tradicionales 7-8 sesiones, a 14-25 (una temporada completa); (2) afiliación: los jóvenes se adhieren a un equipo a lo largo de toda la temporada, que debe ser larga, potenciando y favoreciendo el desarrollo social, el trabajo en equipo y las habilidades sociales; (3) competición regular: está presente de forma constante, pues en el modelo se combinan cíclicamente la competición con otro tipo de ejercicios concretos orientados a desarrollar algún tipo de habilidad; (4) registros de datos: son sistemáticos y variados (valoraciones de los jueces, puntuaciones de los equipos, registros de pases o recepciones, etc.), ya que son necesarios para proporcionar un feedback muy útil a los alumnos que les oriente 
sobre su situación de aprendizaje y los implique en la misma; (5) evento final: se da cabida a una competición final (evento culminante) y su pertinente festividad final como cierre de toda la temporada, nombrando a los equipos vencedores y a aquellos jugadores que más han destacado en diversos tipos de comportamientos y (6) festividad: la temporada debe de ser festiva, tanto alumnos como profesores tienen que hacer lo posible para garantizar un ambiente festivo donde se celebren los éxitos y logros obtenidos por los diferentes equipos y estudiantes. Finalmente, otra cuestión importante dentro del MED es que los alumnos ejercen diferentes roles (preparador físico, entrenador, juez árbitro, preparador físico, etc.) que potencian su autonomía, responsabilidad y les proporcionan experiencias desde diversas dimensiones que ayudan a potenciar su aprendizaje. El MED ha sido estudiado profusamente desde diversas ópticas en los últimos veinte años, tanto fuera (e.g. Hastie, 1996; Hastie y Sinelnikov, 2006; Farias, Mesquita y Hastie, 2015; Pereira et al., 2015) como dentro de nuestro país (e.g. Calderón, Martínez de Ojeda, Martínez, 2013; Cuevas, García y Contreras, 2015; García, Gutiérrez, González y Valero, 2012; Gutiérrez, García, Chaparro y Fernández, 2014; Mahedero, Calderón, Arias-Estero, Hastie y Guarino, 2015; Meroño, Calderón y Hastie, 2015).

Hastie (1996) examinó la participación de alumnos de 6 de Primaria a través de una temporada de speedball y el MED. Los resultados mostraron un alto nivel de participación por parte de los alumnos tanto en el juego como en los roles no participativos. Hastie y Sinelnikov (2006) reportaron resultados positivos de motivación, participación, disfrute además de competencia en roles como el coach en una temporada de baloncesto con alumnos de $6^{\circ}$ de Primaria. Farias et al. (2015) hibridaron el MED y el Modelo de Competencia en deportes de invasión en una temporada de fútbol para analizar el rendimiento y comprensión del juego en alumnos de $5^{\circ}$ de Primaria. Los resultados señalaron una mejora en el rendimiento y comprensión del juego tras experimentar el modelo híbrido. Pereira et al. (2015) examinaron el rendimiento técnico en tres modalidades de atletismo: carrera de vallas, lanzamiento de peso y salto de longitud a través del MED y en comparación con un grupo control basado en la instrucción directa. Los resultados mostraron una mejora en las tres disciplinas tras haber experimentado el MED. Los participantes que siguieron un enfoque basado en la instrucción directa solo reportaron mejoras en chicos con alto nivel de competencia motriz. Calderón et al. (2013) reportaron una mejora de la autonomía, toma de decisiones y actitudes positivas hacia la EF tras experimentar una UD de bádminton en alumnos de $4^{\circ}$ de la ESO bajo el MED. Cuevas et al. (2015) mostraron mejoras significativas en la satisfacción de competencia tras una implementación de una UD de voleibol bajo las premisas del MED en comparación con un grupo control basado en el modelo tradicional.

García et al. (2012) obtuvieron mejoras significativas en la agresividad dependiendo del rol desempeñado, en el amigo positivo entre los integrantes del mismo equipo, en la disminución del amigo negativo en el caso de las chicas y en la disminución de las conductas pasivas en el total del grupo. Gutiérrez et al. (2014) analizaron la viabilidad del MED en $2^{\circ}$ de Primaria a través de una UD de balón prisionero. Los resultados obtenidos reflejaron percepciones muy positivas tanto por parte de los alumnos como de la profesora que implementó la UD y la observadora externa. Por su parte, en el trabajo de Mahedero et al. (2015) los resultados mostraron que los componentes del rendimiento, toma de decisiones y juego mejoraron tras experimentar una UD de mini-voleibol basada en el MED. Meroño et al. (2015) mostraron en nadadores federados un incremento en el grado de disfrute, soporte de la autonomía, competencia percibida y grado de compromiso tras una intervención bajo el MED. Con todo ello, los resultados obtenidos amparan un modelo que potencia y desarrolla elementos esenciales buscados no solo en la EF sino en todos los objetivos curriculares educativos: participación, autonomía, motivación, disfrute, competencia motora, toma de decisiones, comprensión del juego o grado de compromiso, entre otros.

Por su parte, el Modelo de Responsabilidad Personal y Social (TPSR; Hellison, 2011) nace en la década de los setenta con el principal objetivo de fomentar un desarrollo positivo de jóvenes en situaciones de riesgo de exclusión social, proporcionando experiencias sólidas que ayuden a adquirir una serie de valores y responsabilidades útiles para el trascurso de sus vidas. Los valores fundamentales del TPSR se materializan en la práctica a través de una serie de niveles de responsabilidad que los alumnos poco a poco van tratando de conseguir en forma de metas. Los niveles comienzan en el 0, que corresponde a la irresponsabilidad de la que partían los jóvenes a los que se dirigía el programa. Seguidamente, los alumnos tratan de alcanzar los siguientes cinco niveles: (1) respeto por los derechos y opiniones de los demás, (2) participación y esfuerzo, (3) autonomía, (4) ayuda y liderazgo, y finalmente, (5) transferencia. Los niveles 1 y 4 corresponden con la responsabilidad social mientras que los niveles 3 y 4 con la responsabilidad personal. A partir del desarrollo de ambas responsabilidades se puede alcanzar el nivel 5, el más complejo de todos: la transferencia a otros contextos. A partir de cada uno de los niveles se ramifican una serie de componentes que se han de trabajar a lo largo de la puesta en práctica del TPSR. Al nivel 1 le corresponden componentes como el auto-control o la resolución de problemas de forma pacífica; al nivel 2 la automotivación y la capacidad de perseverarse; al nivel 3 la capacidad para trabajar independientemente y el coraje para resistir la presión de grupo; el nivel 4 lo forman, entre otros, la ayuda y comprensión, así como la fuerza interior. Finalmente, el nivel 5 lo componen elementos como la transferencia a otros contextos y el ser un modelo positivo para el resto de personas, especialmente los más jóvenes (Hellison, 2011). Por otra parte, Hellison (2011) propone diferentes estrategias para resolver problemas y situaciones específicas. Algunas de ellas son la autoreflexión, el principio del acordeón, progresiva separación del grupo, o el banquillo del diálogo. Además, para fomentar el aprendizaje y desarrollo de la responsabilidad personal y social en los estudiantes también utilizan otras estrategias como hacer equipos, modificar las tareas, plan personal de trabajo u objetivos en grupo, entre otros.

La educación en valores es fundamental en el ámbito educativo y a ello puede contribuir significativamente la EF (Venero, 2007). EITPSR ha sido objeto de investigación desde su creación en la década de los setenta, aunque en los últimos quince años ha habido una mayor proliferación de trabajos tanto de carácter nacional (e.g. Caballero, 2012, 2015; Carreres, 2014; Escartí, Gutiérrez, Pascual, Marín, Martínez y Chacón, 2006; Marín, 2011) como internacional (e.g. Buchanan, 2001; Caballero, Delgado y Escartí, 2013; Martinek y Hellison, 1997). Caballero (2012) analizó la implementación de una intervención basada en el TPSR en alumnos del ciclo formativo de conducción de actividades físico-deportivas en el medio natural. Los resultados mostraron que en variables como la responsabilidad, empatía, conductas prosociales y clima social de aula, los estudiantes del grupo experimental mostraron significativamente mayores niveles que los de un grupo control que no utilizó el TPSR. Asimismo, la percepción del alumnado y profesorado sobre el programa fue muy positiva, manifestando mejoras en la responsabilidad y cualificación profesional, entre otras. La utilización de actividades en el medio natural como vehículo para potenciar la responsabilidad personal y social fue muy positiva. Carreres (2014) analizó la implementación de un programa basado en el TPSR en adolescentes que practicaban fútbol a nivel extraescolar. Los resultados obtenidos en las variables de responsabilidad personal y social, conducta prosocial y autoeficacia señalaron que tras la experiencia, el grupo experimental, que se implementó bajo el TPSR, presentó mayores niveles en las variables que el grupo control que no experimentó el TPSR.

Escartí et al. (2006) aplicaron una intervención bajo las premisas del TPSR en clases de EF impartidas a adolescentes en riesgo. Los resultados señalaron una reducción en las conductas agresivas y de interrupción además de una positiva evaluación del programa para fomentar la responsabilidad. Por su parte, Marín(2011) encontró diferencias significativas en autoeficacia y autoregulación en alumnos de $5^{\circ}$ de Primaria tras experimentar un programa basado en el TPSR en comparación con un grupo control. Buchanan (2011) analizó la implementación del TPSR en adolescentes en riesgo que asistieron a un campamento deportivo. Los resultados derivados de las entrevistas y las observacio- 
nes reflejaron al TPSR como un instrumento útil para fomentar la asunción de responsabilidades, autoconocimiento y mejorar la disciplina. El trabajo de Caballero et al. (2013) realiza una completa revisión de los estudios relacionados con el TPSR en España y Estados Unidos. Los resultados de estos trabajos han evidenciado la utilidad de este modelo a la hora de desarrollar la responsabilidad personal y social y aquellos componentes interrelacionados con ellas: empatía, conductas prosociales, clima de aula positivo, autoeficacia, reducción de conductas agresivas y de interrupción, autoregulación o autoconocimiento.

Las hibridaciones de modelos de enseñanza pueden ser opciones muy interesantes en el campo educativo, pues permiten abordar los procesos de enseñanza-aprendizaje desde una dimensión bilateral en la que ambos modelos se complementen, creando una especie de simbiosis que permitan a los discentes beneficiarse de las ventajas que cada modelo puede acarrear. Tal como afirman Joyce y Weil (1985, p. 11) «(...) no existe ningún modelo capaz de hacer frente a todos los tipos y estilos de aprendizaje, no debemos limitar nuestros métodos a un modelo único, por atractivo que sea a primera vista». La hibridación de modelos pedagógicos ayuda a incrementar las potencialidades que los diferentes modelos tienen por sí solos, permite llegar de una manera más real y directa a todo tipo de estudiantes (Fernández-Río, 2014), produciendo una estructura mucho más efectiva para docentes y discentes (Fernández-Río, 2015).

En la actual literatura científica solo se conoce un trabajo que haya hibridado el MED y el TPSR. Los autores de este trabajo propusieron un nuevo modelo fruto de la hibridación que denominaron bajo el nombre de Empowering Sport cuyo objetivo es desarrollar por igual el fortalecimiento de roles, la responsabilidad personal y social y la potenciación de habilidades deportivas (Hastie y Buchanan, 2000). Pese a que solo exista este estudio sobre cómo se hibrida el MED y el TPSR, sí que hay publicaciones que han señalado la necesidad de que para que el MED se convierta en un modelo que consiga resultados positivos en el desarrollo de la responsabilidad es necesario que en el mismo se apliquen estrategias específicas actitudinales, muchas de ellas coincidentes con las del TPSR (García y Gutiérrez, 2015; García et al., 2012; Harvey, Kirk y O’Donovan, 2014). En el ámbito de las artes marciales y deportes de combate (AAMM y DDCC) se desconocen trabajos publicados que hayan utilizado este contenido bajo la premisa del MED. Sí que existen una serie de trabajos que han implementado el TPSR a través de las artes marciales como contenido para favorecer el desarrollo de habilidades cognitivas, socioafectivas y motrices (Wright, 2001, 2002; Wright y Burton, 2008; Wright, Dyson y Moten, 2012; Wright, Li, Ding y Pickering, 2010; Wright, White y Gaebrel-Spira, 2004).

El kickboxing es un deporte de combate en el que se emplean técnicas de puño propias del boxeo con otras de pierna más características de otros deportes como el karate o el taekwondo. Está formado por siete disciplinas: tres de contacto pleno (Full Contact, K1 y Low Kick), tres de contacto controlado (Light Contact, Kick Light y Point Fighting) y una de ellas sin contacto (formas musicales o Musical Forms) (WAKO, 2011). Educativamente hablando, destaca la última de ellas pues es la que se utilizó predominantemente en la experiencia que se presenta en este trabajo. «Una forma musical es una pelea imaginaria contra uno o más oponentes en la que el competidor utiliza técnicas procedentes de Artes Marciales Orientales específicamente adaptadas a la música elegida. La elección de la música es personal» (WAKO, 2011, p. 3). No obstante, en el ámbito escolar, tan solo se tiene constancia de dos trabajos publicados que han utilizado el kickboxing como contenido educativo (Menéndez y Fernández-Río, 2014a, 2014b). Sin embargo, estos trabajos no se implementaron en Educación Secundaria Obligatoria(ESO), ni a través de cualquiera de los dos modelos pedagógicos que aquí se presentan. Teniendo en cuenta que, como anteriormente se mencionó, es necesario incluir elementos relativos al TPSR en el MED para poder fomentar conductas y actitudes de responsabilidad, a lo que se suman los beneficios que pueden tener las AAMM y DDCC en adolescentes y niños (ver revisión Vertonghen y Theeboom, 2010), fue importante testar este modelo híbrido a través de un contenido innovador como el kickboxing. Por lo tanto, el objetivo fundamental de este trabajo fue el de introducir el kickboxing como contenido educativo a través de un modelo híbrido (MED y TPSR) en alumnos de ESO.

\section{Planteamiento didáctico de la experiencia}

\section{Contextualización}

La unidad didáctica(UD) desarrollada se introdujo en el $2^{\circ}$ ciclo de la ESO ( $4^{\circ}$ curso). Se implementó durante los meses de enero, febrero y marzo del año 2015 a lo largo de 16 sesiones (2 por semana) con una duración de 55 minutos cada una de ellas. Todas las sesiones se impartieron en el gimnasio. En la experiencia participaron un total de 78 alumnos (cinco grupos naturales) con edades comprendidas entre los 15 y 17 años (M=15.4 \pm .73$)$. Ni los alumnos participantes ni el docente que aplicó la intervención tenían experiencia previa con los modelos implementados.

\section{Objetivos didácticos}

La legislación utilizada para el desarrollo de la UD fueel Decreto 74/ 2007, de 14 de junio, por el que se regula la ordenación y establece el currículo de la Educación Secundaria obligatoria en el Principado de Asturias (Decreto, 74/2007). Los objetivos planteados fueron:

- Practicar un contenidonovedoso en el currículo como el kickboxing que ayude a que los jóvenes descubran y exploren nuevos movimientos y gestos deportivos que potencien su riqueza motriz y dominio corporal.

- Favorecer el diseño y práctica de actividades rítmico-expresivomusicales como vehículo para aprender a comunicar y transmitir con el movimiento corporal.

- Utilizar el kickboxing como actividad que favorece el desarrollo de habilidades como la agilidad y cualidades motrices como la coordinación dinámica general, segmentaria, equilibrios, golpeos o desplazamientos.

- Potenciar conductas de responsabilidad personal y social que favorezcan el desarrollo de habilidades y actitudes como la empatía, el trabajo en equipo, el respeto por las opiniones de los demás, la autonomía o la participación.

\section{Contenidos}

La UD se incluyó dentro de los bloques de contenidos 3: Juegos y deportes y 5: Expresión corporal. Los contenidos específicos desarrollos fueron:

\section{- Detipo conceptual}

- Técnicas básicas puño y pierna: directo, gancho, croché, frontal, circular y lateral.

- Técnicas básicas de defensa y desplazamientos.

- De tipo procedimental

- Golpeos al aire y dianas fijas y móviles de diferente tamaño y material, en el suelo o en altura, estática o dinámicamente.

- Actividades coreográficas o de expresión corporal en grupo. - De tipo actitudinal

- Respeto, participación, esfuerzo, autonomía, ayuda y liderazgo.

\section{Competencias básicas}

La evolución constante que ha sufrido nuestra sociedad demanda cambios en materia curricular con el objetivo de formar ciudadanos plenos. En ese sentido, el Marco Europeo implantó una serie de aprendizajes necesarios que deben obtenerse a lo largo de la escolaridad obligatoria como instrumentos para adaptarse correctamente a la ciudadanía moral y democrática

La EF potencia de una forma u otra las ocho competencias básicas expuestas en la Ley Orgánica 2/2006, de 3 de mayo, de educación (Ley Orgánica 2/2006). No obstante, en la presente propuesta solo mencionaremos aquellas competencias más desarrolladas en esta UD:

- Competencia cultural y artística: fomentar el disfrute y aprecio de manifestaciones deportivas minoritarias como el kickboxing como 
elemento para escapar de la oligarquía deportiva presente en nuestra sociedad, así como elemento para el crecimiento cultural y artístico. También como forma de adquirir un pensamiento reflexivo sobre dichas manifestaciones que nos permita adentrarnos en una sociedad estereotipada con un mayor bagaje crítico.

- Competencia social y ciudadana: el MED y el TPSR posibilitan el fomento de la participación tanto activa y autónoma, como grupal y cooperativa. El kickboxing como vehículo para desarrollar las habilidades de liderazgo, trabajo en equipo, respeto y ayuda a los demás que estos modelos potencian y reclaman.

- Competencia en el tratamiento de la información y competencia digital: el MED y el MRSP fomentan las habilidades para manejar medios audiovisuales que permita a los alumnos utilizarlos como medios de comunicación y trabajo cooperativo. También como elementos para realizar evaluaciones de los procesos de enseñanzaaprendizaje del alumnado a través de las nuevas tecnologías de la información

- Autonomía e iniciativa personal: la autonomía como uno de los ejes principales del MED y el TPSR que posibilitan la organización, creación y puesta en práctica de actividades autónomas sin la supervisión directa del profesor. La adquisición de roles que fomentan las habilidades de responsabilidad, iniciativa personal y liderazgo hacia la consecución de metas a través de la perseverancia y la superación.

\section{Diseño}

Para estudiar la validez del programa aplicado, se utilizaron las recomendaciones de Hastie y Casey (2014, p. 423): «a) una rica descripción de los elementos curriculares de la unidad, b) una detallada validación del modelo implementado y c) una detallada descripción del contexto del programa». Por ello, para potenciar la hibridación de estos enfoques se utilizaron tanto aspectos clave del MED (temporada, afiliación, competición regular, registros de datos, evento final, festividad y roles) como del TPSR (estructura de la sesión, puesta en práctica de los niveles de responsabilidad y estrategias para resolver problemas y situaciones específicas). A continuación explicamos cómo se han integrado tanto los elementos del MED como los del TPSR en esta experiencia:

\section{MED}

\section{Temporada}

La temporada fue en forma de campeonato y siguió un diseño de carácter progresivo (Siedentop et al. 2011) con dos fases competitivas. Se denominó «Campeonato del Mundo de Kickboxing Educativo» y fue un torneo donde participaron los 5 grupos naturales de 4 de la ESO (19 equipos). El campeonato tuvo una duración de 16 sesiones (2 por semana). La primera sesión fue de carácter explicativa en ella se enseñó

Tabla 1 .

Plan de actividades de la UD de kickboxing educativo.

\begin{tabular}{|c|c|}
\hline \multicolumn{2}{|c|}{ Sesión } \\
\hline 1 & $\begin{array}{l}\text { - Introducción al MED y al TPSR: roles diarios, equipos, selección del país, niveles } \\
\text { de responsabilidad. }\end{array}$ \\
\hline $2-4$ & $\begin{array}{l}\text { - Habilidades técnicas básicas: jab y cruzado, patada frontal y desplazamientos; } \\
\text { croché, patada circular y técnicas de defensa; gancho, patada lateral y } \\
\text { combinaciones. } \\
\text { - Objetivos comportamentales: no insultar o reírse de otros compañeros (sesión n²), } \\
\text { autocontrolar el temperamento (sesión no } 3 \text { y esforzarse en las tareas y actividades } \\
\text { que menos gustan (sesión no } 4 \text { ). }\end{array}$ \\
\hline 5 & $\begin{array}{l}\text { - Desfile de países e himnos nacionales. } \\
\text { Objetivo comportamental: mantener una buena relación con los compañeros de } \\
\text { equipo. }\end{array}$ \\
\hline 6-8 & $\begin{array}{l}\text { - Repaso de habilidades. } \\
\text { - Fase competitiva 1: técnicas básicas de kickboxing. } \\
\text { Objetivos comportamentales: participar sin la supervisión del profesor (sesión } \\
\mathrm{n}^{\circ} 6 \text { ), proponer y tratar de cumplir una meta concreta individual (sesión n7 y ser } \\
\text { capaz de automotivarse (sesión nº). }\end{array}$ \\
\hline 9-14 & 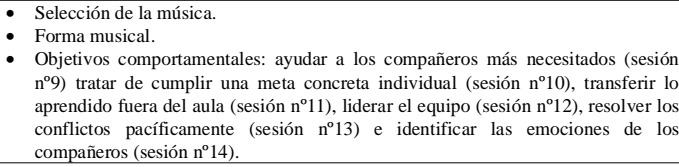 \\
\hline 15 & $\begin{array}{l}\text { - Ensayo previo. } \\
\text { - Fase competitiva 2: formas musicales, Evento Culminante. } \\
\text { - Objetivo comportamental: no reírse de los fallos de los compañeros. }\end{array}$ \\
\hline 16 & $\begin{array}{l}\text { - Entrega de premios: Festividad. } \\
\text { - Objetivo comportamental: llevarse bien con los compañeros de otras clases. }\end{array}$ \\
\hline
\end{tabular}

Tabla 2 .

Clasificación de los puntos.

CLASIFICACIÓN DE LOS PUNTOS DEL CAMPEONATO DEL MUNDO DE KICKBOXING

\begin{tabular}{llc}
\hline Puntos de competición & $\cdot$ Técnicas de kickboxing & 15 \\
\hline Puntos por deportividad & $\cdot$ Formas musicales & 15 \\
\hline Puntos de equipo & & 15 \\
\hline \multirow{2}{*}{ Puntos cognitivos } & $\cdot$ Webquest & 30 \\
\hline & $\cdot$ Cumplimiento rol de juez & 15 \\
& Total puntos & 10 \\
\hline
\end{tabular}

Tabla 3

Escala de valoración de técnicas de kickboxing.

País del juez : $\quad$ Deportista: $\quad$ Ronda $^{\circ}$ :

Velocidad (hasta 3 puntos)

Precisión (hasta 3 puntos)

Calidad técnica (hasta 6 puntos)

Flexibilidad (hasta 3 puntos)

Puntuación total:

Tabla 4 .

Tablón de resultados competitivo

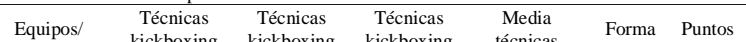

\begin{tabular}{|c|c|c|}
\hline Competición & kickboxing & kickboxing \\
\hline
\end{tabular}

\begin{tabular}{|c|c|c|c|c|c|}
\hline & (Ronda 1) & (Ronda 2) & (Ronda 3) & kickboxing & \\
\hline Equipo $\mathrm{A}$ & Puntos & Puntos & Puntos & Puntos & P \\
\hline
\end{tabular}

1.

3.

Media puntos

a los alumnos las principales características de ambos modelos y se formaron los equipos. En las sesiones 2, 3y 4, tuvo lugar la pretemporada. Cada equipo aprendió las técnicas básicas del kickboxing en su territorio particular del gimnasio guiado por el profesor, bien golpeando al aire, a un churro de espuma realizando actividades de desplazamiento y defensa entre compañeros. En la sesión 5 se realizó el desfile de países donde sonaron los himnos nacionales y posteriormente se procedió a explicar a los alumnos cómo se iban a desarrollar las fases competitivas (jueces, coach, sistema de puntuación etc.). En las sesiones 6, 7 y 8 se llevó a cabo la fase competitiva 1: técnicas básicas de kickboxing. A continuación comenzó la fase competitiva 2: formas musicales. De la sesión 9 a la 14 se diseñaron y perfeccionaron las formas musicales. En la sesión 15 tuvo lugar en el evento culminante en el que todos los equipos expusieron su coreografía. Finalmente, la festividad del campeonato se celebró en la sesión 16.

\section{Afiliación}

En la primera sesión se procedió a seleccionar libremente los países que cada equipo tendría que representar a lo largo del campeonato. Para formar los equipos, se seleccionó cuatro alumnos/as que ejercieron de capitanes y estos/as tuvieron que escoger compañeros siguiendo el orden de chico-chica para que los grupos fueran lo más heterogéneos posibles. Además, para fomentar la afiliación grupal, cada equipo tuvo que crear camisetas y banderas autoconstruidas, inventar un saludo y descargar el himno nacional de cara al desfile de países.

Competición regular, registro de datos y evento culminante

Como se mencionó anteriormente, el campeonato siguió un diseño progresivo con dos fases competitivas en el que los cinco grupos naturales de $4^{\circ}$ de la ESO compitieron entre ellos. Para ganar el campeonato, los equipos tuvieron que obtener la máxima puntuación posible en varios apartados (Tabla 2).

(1) Puntos de competición: dos apartados:

a) Técnicas de kickboxing: los encargados del material de cada equipo montaban la zona de competición en una esquina del gimnasio, compuesta por cuatro colchonetas y un banco sueco pegado a cada una de los cuatro lados de las colchonetas, donde se sentaban los jueces (1 por equipo) con su acreditación de juez, la escala de valoración de técnicas de kickboxing (Tabla 3) y una rúbrica para orientarse a la hora de calificar a sus compañeros. El alumno ejecutante, tras ser llamado por uno de los jueces, acudía junto con su coach a esta zona de competición, saludando a los jueces. Una vez allí extraía de una bolsa una combinación de movimientos, por ejemplo: cinta, desplazamiento hacia adelante, directo de 1, directo de 2 y patada frontal de 2 (ver aprendizaje por claves más abajo para clarificar las claves de «1» $\mathrm{y} « 2 »)$. Los jueces daban, como máximo, 30 segundos para que el alumno ejecutase la 
Tabla 5.

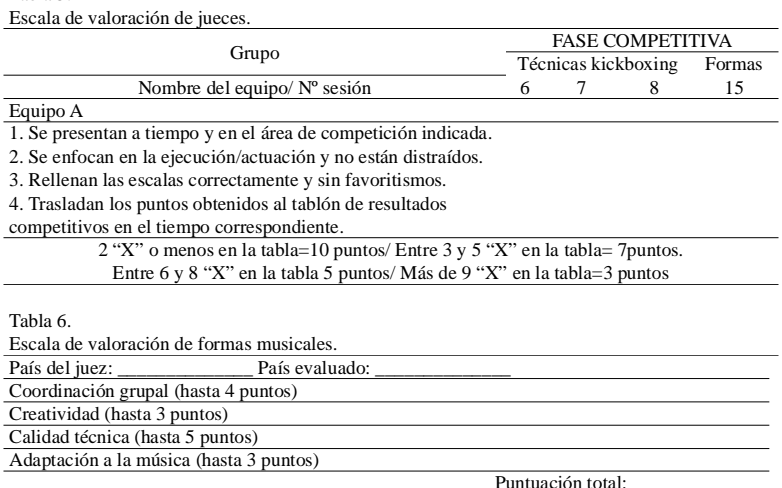

técnica sobre un churro de espuma que tenía una cinta negra pegada en la parte superior para poder calificar la precisión de los golpes. Este churro era sujetado por su coach que debía mantenerse en silencio en todo momento. En caso de superar los 30 segundos o hablar entre coach y competidor, los jueces valoraban con 0 puntos la ejecución. Una vez realizada esta, los jueces cumplimentaban individualmente la escala de valoración con un máximo de hasta 15 puntos. A continuación, se llamaba al siguiente competidor hasta completar la ronda/sesión (7-8 alumnos por ronda/sesión). Finalmente, los jueces se reunían y hacían una media de las puntuaciones otorgadas a cada uno de los alumnos que compitieron esa sesión. Posteriormente, los jueces tenían que trasladar las puntuaciones al tablón de resultados competitivos (Tabla 4). Desde que empezaban a llamar a los competidores hasta que finalizaban el proceso trasladando los puntos disponían de un máximo de 20 minutos. En el caso de superar ese tiempo, el profesor anotaba una X en el ítem nº 4 de la escala de valoración de jueces (Tabla 5). Esto podía terminar en la no obtención de puntos por adecuado desempeño del rol de juez (ver puntos cognitivos más abajo). Al final de la fase competitiva 1 , todos los alumnos debían haber participado en la competición de técnicas de kickboxing.

b) Formas musicales (evento culminante): siguió un procedimiento similar. La zona de competición esta vez se montó en el centro del gimnasio y los jueces utilizaron la escala de valoración de formas musicales (Tabla 6) y su pertinente rúbrica para orientar la calificación. En esta fase competitiva, el resto de equipos de la clase actuaban como espectadores, restando puntos de deportividad en el caso de que hubiera comportamientos inadecuados. Para obtener la puntuación final de las fases competitivas, tan solo había que sumar los hasta 15 puntos máximos obtenibles en la fase 1 de técnicas básicas y los 15 máximos en la fase 2 de formas musicales.

(2) Puntos de deportividad: todos los equipos partían con 15 puntos y el profesor restaba puntos en el caso de que algún equipo mostrase algunas de las conductas antideportivas señaladas en la escala de depor-

Tabla 7.

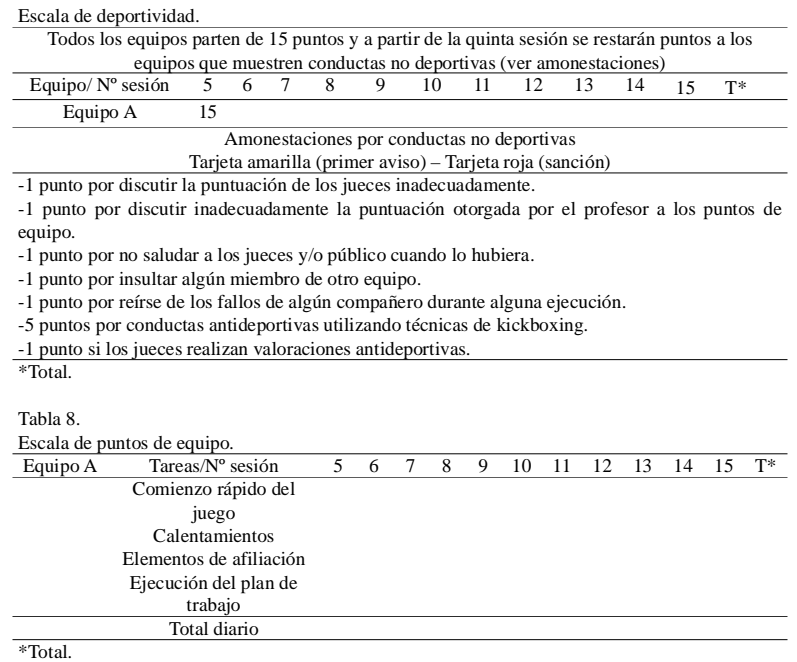

tividad (Tabla 7). Además del profesor, los jueces también tenían potestad para informar al docente de conductas no deportivas mostradas por alguno de los alumnos mientras ejecutaban las combinaciones en la zona de competición. Esta escala se utilizó desde la sesión 5 hasta la sesión 15.

(3) Puntos de equipo: el docente utilizó la escala de puntos de equipo (Tabla 8) para valorar cuatro cuestiones: calentamientos (el equipo sigue el plan de calentamiento de forma ordenada, realiza los ejercicios correctamente, se ajusta al tiempo estipulado y el preparador asume el rol de líder), inicio rápido del juego (los alumnos tardan menos de cinco minutos desde que suena la campana hasta que se sitúan en equipo alrededor del profesor), elementos de afiliación: bandera, himno nacional, indumentaria y saludo preparado (todos los miembros del equipo traen todos los elementos de afiliación preparados), ejecución plan de trabajo (el equipo realiza correctamente las actividades señaladas en el plan de trabajo, se ajusta al tiempo estipulado y el plan está dirigido correctamente por el coach/coreógrafo). Esta escala se utilizó desde la sesión 5 hasta la sesión 15.

(4)Puntos cognitivos: delos 25posibles, 15procedíandela WebQuest que los alumnos tuvieron que elaborar y enviar al docente (1 punto por pregunta; ver evaluación conceptual más abajo en el apartado «evaluación y criterios de evaluación»). Para obtener la puntuación final del equipo se realizó la media de todas las puntuaciones obtenidas por cada uno de los integrantes del equipo en esta WebQuest. Si algún alumno no entregaba la WebQuest se valoraba con un 0 puntos su trabajo. Los restantes 10 puntos procedían del adecuado cumplimiento del rol de juez (Tabla 4). Esta escala se utilizó en las sesiones que correspondían a las 2 fases competitivas de este campeonato: sesiones 6, 7, 8 y 15.

De forma extraordinaria, se invitó a los estudiantes a que elaborasen un trofeo autoconstruido para entregar en la festividad final a un alumno a título individual o a un equipo por las razones que ellos considerasen (ver Figura 1). La realización de este trofeo se valoró con +2 puntos y en todo momento se informó a los estudiantes de que su elaboración solo contaba para los puntos del campeonato y no para la calificación de la asignatura.

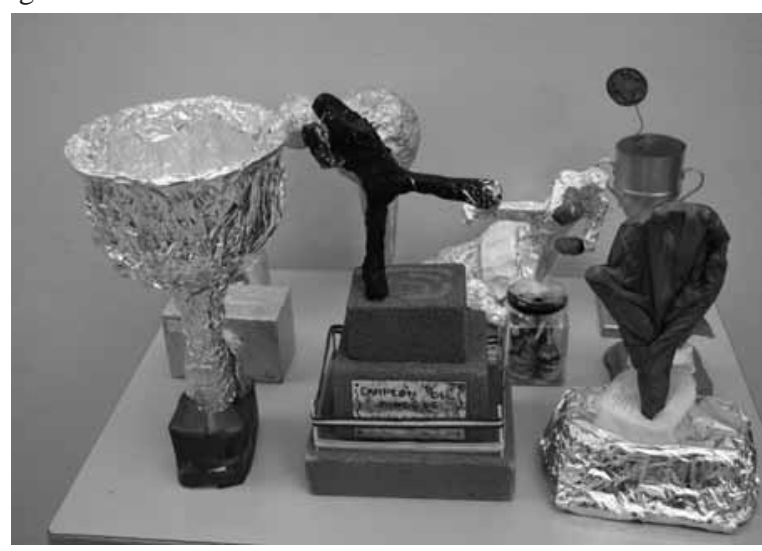

Figura 1. Trofeos autoconstruidos.

Festividad

Para generar un ambiente festivo se utilizaron los elementos de afiliación (camisetas y banderas autoconstruidas, selección de un país, invención de un saludo) y se realizó un desfile de países con el himno nacional de cada uno de ellos. Además, al final del campeonato, en la última sesión, se celebró una fiesta de clausura en la que se juntaron en el gimnasio todos los alumnos que participaron en el campeonato para la entrega de premios. Los premios fueron de diversa índole: primer, segundo y tercer puesto; equipo más deportivo; equipo más competitivo; equipo que sacó mejores fotografías; equipo más afiliado o premio al equipo que presentó la mejor coreografía. Los premios fueron entregados en forma de diplomas plastificados. A esto se le suma los trofeos autoconstruidos que se citaron anteriormente y que cada equipo opcionalmente dio a algún equipo o persona por las razones que ellos consideraron. Tras la entrega de premios hubo una comida amenizada por música que el propio alumnado seleccionó. 
Roles

Los empleados en esta UD fueron los siguientes: (1) coach: se encargó principalmente de motivar y liderar el equipo, así como de llevar a cabo el «plan de trabajo» que cada equipo tuvo que realizar a partir de la sexta sesión; (2) encargado del material: se encargó de sacar y devolver a su lugar correspondiente el material utilizado por el equipo (a excepción de las colchonetas), informar de cualquier rotura del mismo y encargarse del sistema de sonido del equipo para las formas musicales; (3) preparador físico: encargado de dirigir los calentamientos utilizando el plan de calentamiento que cada equipo tenía; (4) fotógrafo: realizó capturas fotográficas diarias de lo más significativo que aconteció en su equipo a lo largo de la experiencia; (5) coreógrafo: encargado de coordinar y dirigir el diseño de la forma musical del grupo y ayudar al resto del equipo a aprender las secuencias de los movimientos utilizando el plan de trabajo; y (6) juez: se encargaba de valorar las ejecuciones técnicas y las formas musicales de cada equipo a través de una plantilla de puntuación (Tablas 3 y 6) y con la ayuda de una rúbrica que era pública para que todos supieran en qué criterios basarse para dar una u otra puntuación. También se encargaban de trasladar las puntuaciones obtenidas por cada alumno/equipo al tablón de resultados. Todos los roles fueron rotativos; se cambiaban cada sesión. Para el correcto aprendizaje de los mismos, se introdujeron de forma progresiva. En la segunda sesión se introdujeron los roles de fotógrafo y preparador físico, en la tercera sesión el encargado del material, en la cuarta sesión el de coach, en la quinta sesión el de juez y finalmente, en la novena sesión, el rol de coreógrafo.

\section{TPSR}

\section{Estructura de la sesión}

La estructura de todas las sesiones siguió el esquema propuesto por Hellison (2011) para fomentar la adquisición de la responsabilidad personal y social: (1) tiempo de relación: en el que el profesor, bien antes o después de las sesiones, interactuó con los alumnos para fomentar su relación; (2) toma de conciencia previa: en la que a los alumnos, tras llegar al gimnasio, se les explicaba cuáles eran los tipos de comportamientos que los jóvenes tenían que mostrar en la sesión, directamente relacionados con algunos de los niveles de responsabilidad; (3) responsabilidad en acción: donde, bien de forma grupal en las primeras sesiones, o por equipos en la parte principal del campeonato, los alumnos tenían que poner esos comportamientos en acción a través de la actividad física; (4) encuentro de grupo: en el que los jóvenes se sentaban en círculo con el profesor y compartían opiniones y sentimientos acontecidos durante esa sesión y (5) autoevaluación y evaluación: en la que los estudiantes individualmente valoraban sus comportamientos y actitudes asignándose una calificación ente el 0 y el 10. El profesor también evaluaba a sus estudiantes con el mismo método para posteriormente extraer la nota comportamental (ver «evaluación y criterios de calificación» abajo) y orientar a los alumnos hacia una evaluación crítica y justa. También se evaluaba la sesión grupalmente en base al objetivo comportamental relacionado con los niveles de responsabilidad que se planteaba en la toma de conciencia.

\section{Puesta en práctica de los niveles de responsabilidad}

Los niveles de responsabilidad fue un elemento que tuvo una importancia muy significativa dentro de la experiencia. Se pegó a la pared del gimnasio una gran cartulina con los niveles de responsabilidad dibujados. El primer día se explicó a los alumnos cómo se abordaría el trabajo con dichos niveles para posteriormente ponerlos en práctica (responsabilidad en acción). Como se vio en el apartado anterior, en la toma de conciencia se explicaba al alumnado el objetivo comportamental que se iba a trabajar en cada una de las sesiones, siempre teniendo en cuenta que cada objetivo estaba relacionado con alguno de los niveles de responsabilidad (ver objetivos comportamentales en Tabla 1). Se comunicó que se trabajarían de forma ascendente aunque en algunos momentos algunos alumnos, equipos o incluso todo el grupo podían estar bien por encima o por debajo del nivel que en cada sesión se trabajaba. También se informó que en muchas ocasiones se volvería a trabajar niveles de responsabilidad inferiores aunque el alumnado estuviera avanzado en dichos niveles (por ejemplo, en la última sesión se trabajó el nivel 1). Al final de la sesión, en la evaluación, todo el grupo evaluaba la sesión en base al objetivo comportamental que se planteaba en la toma de conciencia utilizando el dedo pulgar (hacia arriba, positivo; horizontal, neutro, hacia abajo, negativo).

\section{Estrategias para resolver problemas y situaciones específicas}

El profesor utilizó diversas estrategias para resolver problemas y situaciones específicas (e.g. resolución de conflictos) basadas en las indicaciones de Hellison (2011). Estas estrategias fueron: para el nivel 1: principio del acordeón, es decir, se aumentó o redujo el nivel de práctica en función de comportamiento mostrado por los alumnos; negociación, es decir, ofrecer a los estudiantes diferentes posibilidades a elegir para poder darles responsabilidad, y el banquillo del diálogo, en el que los alumnos que tuvieron algún tipo de conflicto, se sentaban en un banco del gimnasio para dialogar de forma pacífica y resolver el problema; para el nivel 2: enseñanza por invitación, esto es, invitar a los estudiantes a probar nuevas actividades, comportamientos, habilidades o ejercicios; para el nivel 3 se utilizaron diferentes estrategias para facilitar autonomía: reflexionar en el encuentro del grupo sobre esta cuestión, proponerse metas y utilizar el principio del acordeón para ajustar la cantidad de tiempo destinado a este nivel; para el nivel 4 el profesor propició feedback a los líderes de los grupos para que no fueran demasiado arrogantes, directivos y fomentarán refuerzos positivos que crearan de equipo confortable. Finalmente, para el nivel 5, se propuso como estrategia principal castigar al alumnado que utilizara en forma de agresión las técnicas de kickboxing aprendidas fuera del gimnasio, aunque esto no se tuvo que llevar a cabo a ningún momento de la intervención.

En otro sentido, es importante señalar que la UD se llevó a cabo a través de actividades sin contacto. Las ejecuciones de técnicas siempre se realizaron golpeando al aire o a churros de espuma que otro alumno sujetaba a una distancia lo suficientemente segura como para que no existiera ningún tipo de percance. Además, desde la primera sesión, se dejó clara la orientación pedagógica del kickboxing y el formato del mismo, más orientado hacia lo artístico que hacia lo que inicialmente la mayoría pensaban: un enfoque con contacto. No obstante, los alumnos comprendieron velozmente esta versión diferente que se le otorgó al kickboxing y tanto chicos como chicas se sintieron igual de integrados con este deporte.

En la Tabla 1 se puede ver el plan de actividades de la UD de kickboxing educativo.Asimismo, en este link: https:/www.youtube.com/ watch?v=FWnFNse2a_I se puede ver el vídeo de los mejores momentos del evento culminante de la experiencia (presentación grupal de las formas musicales). En este caso, la grabación se realizó siguiendo las directrices del Comité de Ética de la Universidad de los autores del manuscrito y tras el consentimiento informado del Centro y los padres.

Para la adecuada captación de la información se ha utilizado una estrategia denominada aprendizaje por claves (Fernández-Río, 2006; Fernández-Río y Méndez-Giménez, 2013). Las claves pueden definirse como frases cortas y precisas que remarcan las partes esenciales de la habilidad a aprender, y que pueden ser recordadas fácilmente por los estudiantes en cualquier momento para practicar, guiar, motivar o instruir (Fernández-Río, 2006). Es una estrategia muy significativa en esta UD dado que se trata de un contenido educativo generalmente desconocido por los alumnos, lo que provoca una ausencia de imágenes o referencias mentales que permitan al alumno guiarse en el aprendizaje de forma convincente y segura. En el caso de este campeonato, las principales claves fueron los números «1» y «2» que correspondían a los puños y patadas de izquierda y derecha respectivamente (en el caso de los derechos; lo contrario para los zurdos).

Se llevó a cabo un estudio piloto para testar las actividades y desarrollar la versión final de la intervención (Menéndez y FernándezRío, 2014a). Finalmente, para evaluar la validez del programa, se creó un checklist con ítems correspondientes a ambos modelos (verTabla 9). Se grabaron todas las sesiones, se escogieron cuatro de forma aleatoria y se enviaron a dos expertos en los modelos sin relación alguna con la inves- 
Tabla 9.

\begin{tabular}{|c|c|c|}
\hline \multicolumn{3}{|c|}{$\begin{array}{l}\text { Checklist modelos. } \\
\text { Nombre observador externo: }\end{array}$} \\
\hline \multicolumn{3}{|c|}{ Fecha: Sesión: } \\
\hline \multicolumn{3}{|c|}{$\begin{array}{l}\text { 1. Los estudiantes van a un campo propio y calientan en equipo. } \\
\text { 2. L Los estudiantes practican las habilidades con su equipo en un campo propio. }\end{array}$} \\
\hline \multicolumn{3}{|c|}{$\begin{array}{l}\text { 3. - Los estudiantes siguen roles de trabajo (coach, encargado/s del material, } \\
\text { fotógrafo, preparador físico, coreógrafo). }\end{array}$} \\
\hline \multicolumn{3}{|c|}{ 4. El profesor permite a sus alumnos liderar o estar a cargo de un grupo. } \\
\hline \multicolumn{3}{|c|}{ 5. L Las tareas son mayoritariamente dirigidas por los alumnos. } \\
\hline \multicolumn{3}{|c|}{ 6. El El profesor habla a sus alumnos sobre la responsabilidad personal y social. } \\
\hline \multicolumn{3}{|c|}{$\begin{array}{l}\text { 7. El El profesor proporciona oportunidades para que los alumnos reflexionen, debatan y } \\
\text { compartan ideas en grupo. }\end{array}$} \\
\hline \multicolumn{3}{|c|}{$\begin{array}{l}\text { compartan ideas en grupo. } \\
\text { Nota. Los ítems } 1,2,3 \text { y } 5 \text { están referidos al MED. Los ítems } 4,6 \text { y } 7 \text { están referidos al TPSR }\end{array}$} \\
\hline \multicolumn{3}{|c|}{$\begin{array}{l}\text { Tabla } 10 . \\
\text { Evaluación práctica. }\end{array}$} \\
\hline \multicolumn{3}{|r|}{ UD Kickboxing - Evaluación práctica } \\
\hline \multicolumn{3}{|r|}{ 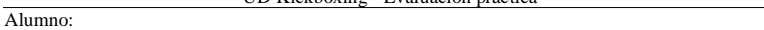 } \\
\hline Sesión & Ítems & Puntuación: Bien=1 - Regular $=0.5-\mathrm{Mal}=0.25$ \\
\hline & 1 & Golpes rectos (jab y cruzado) \\
\hline & 2 & Patada frontal \\
\hline & 3 & Croché \\
\hline & 4 & Patada circular \\
\hline & 5 & Gancho \\
\hline & 6 & Desplazamientos \\
\hline & 7 & Patada lateral \\
\hline & 8 & Técnicas de defensa \\
\hline & 9 & Calidad técnica de la forma musical \\
\hline & 10 & Coordinación grupal de la forma musical \\
\hline & 11 & Creatividad de la forma musical \\
\hline 15 & 12 & Adaptación correcta de la música \\
\hline
\end{tabular}

tigación. Los expertos tenían que cumplimentar en cada una de las sesiones seleccionadas el checklist, escribiendo «Sí» 0 «No» en cada uno de los ítems. Ambos observadores alcanzaron un acuerdo del 100\% de fidelidad en ambos modelos.

\section{Evaluación y criterios de calificación}

Para la evaluación práctica de la UD se utilizó una escala de valoración procedimental (Tabla 10). A partir de este instrumento se evaluaron tanto las técnicas básicas de kickboxing (directo, croché, gancho, frontal, lateral, circular, técnicas de defensa y desplazamientos), como las habilidades trabajadas a través de la forma musical (calidad técnica, coordinación grupal, creatividad y adaptación a la música).

Parala evaluación actitudinal serealizólamediadelas autoevaluaciones de los alumnos y la evaluación del profesor respecto a la actitud mostrada en cada una de las sesiones de la UD de kickboxing en las que se llevaron a cabo estos procedimientos didácticos.

Finalmente, para la evaluación conceptual, los alumnos tuvieron que realizar una WebQuest atendiendo a las necesidades cada vez mayores a nivel escolar de educar a través del uso de las nuevas tecnologías de la información (tratamiento de la información y competencia digital). En la WebQuest, los estudiantes contestaron a 15 preguntas tipo test relacionadas con conceptos trabajados a lo largo de la UD, con apoyo de apuntes teóricos descargados de la propia herramienta. Las cuestiones planteadas se centraron generalmente en la historia del kickboxing, en sus principales características y en las diferentes modalidades que constituyen en este deporte. Además, en la WebQuest se incluyeron tres vídeos de modalidades de contacto controlado (Point Fighting y Light Contact) o sin contacto (formas musicales) para que los alumnos identificaran visualmente las técnicas que algún competidor realizaba. La realización de esta WebQuest permitió que los alumnos obtuviesen una visión más amplia del concepto estereotipado que tenían del kickboxing (una única modalidad de ring y de contacto pleno) y fomentaran su competencia digital. Dos preguntas erróneamente contestadas restaban una correcta. Los criterios de calificación fueron los siguientes: 50\% parte práctica o procedimental, 30\% parte actitudinal (15\% autoevaluaciones de los alumnos y $15 \%$ evaluaciones del profesor) y $20 \%$ por la WebQuest.

\section{Resultados}

Esta experiencia ha puesto de manifiesto los beneficios educativos de la incorporación de modelos pedagógicos al ámbito curricular, en este caso, el MED y el TPSR. El alumnado recibió muy positivamente tanto los enfoques de los modelos como la novedad del deporte, nunca antes practicado por casi ninguno de los participantes. Los resultados en cuanto al diseño de la intervención han sido muy positivos. La competitividad que podía emerger significativamente por el diseño de la experiencia en forma de campeonato fue aplacada por el TPSR, lo que provocó que el alumnado no valorara excesivamente el lugar de la clasificación y sí los valores de responsabilidad personal y social que diariamente se trabajaban. Se creó una atmósfera de trabajo en equipo no competitiva entre los grupos, apoyándose unos a otros en los encuentros grupales finales a través de consejos, sugerencias y aportaciones personales y/o grupales. Los alumnos comprendieron que el objetivo primario de la experiencia no era aprender únicamente kickboxing sino utilizar este contenido para propiciar experiencias deportivas auténticas y fomentar la responsabilidad personal y social.

El diseño con dos fases competitivas (técnicas básicas y formas musicales) fue el apropiado aunque entre las sesiones 6 y 8, durante la competición de técnicas básicas, los alumnos que ese día no competían seguían practicando habilidades técnicas, lo que provocó cierto aburrimiento en el alumnado. Sería interesante para futuras ocasiones que se incluyeran en esas sesiones un trabajo de habilidades motrices (giros, ruedas, volteretas etc.) que posteriormente se pondrían en práctica en la forma musical y así evitar la excesiva practica de habilidades técnicas de kickboxing (desde la sesión n²2 hasta la nº en esta experiencia). Las dificultades del diseño fueron el desconocimiento y puesta en práctica de los modelos, tanto por parte del alumnado como del profesor. En referencia al alumnado, fue complejo que entendieran todas las tablas que se presentaron en la experiencia y el sistema de puntuación. No obstante, con el paso de las sesiones fueron adecuadamente interiorizadas a excepción de los puntos por el correcto desempeño de los jueces. Los alumnos prestaban más atención a los puntos por desempeño técnico y de equipo (Tablas 4 y 8) que a las otras tablas, y al principio tendían a pensar que el resultado final únicamente era el del evento culminante (presentación de las formas musicales), cuando realmente solo representaba 15 puntos de los 100 máximos. Con el paso de las sesiones, el alumnado consiguió comprender que se trataba de una competición de responsabilidad, donde no ganaba el equipo mejor dotado técnicamente sino el más responsable (70 de los 100 puntos eran sobre cuestiones relacionadas con la responsabilidad).

Respecto al profesor, este encontró especialmente difícil la organización de todo el diseño (tablas, sistema de puntuación, enseñanza de los roles, el contenido en sí mismo a nivel motor y la orientación hacia la responsabilidad del campeonato). Quizá un menor número de roles y un sistema de puntos más sencillo hubiera sido una solución para reducir el nivel de exigencia de aplicación del modelo. Con respecto a los roles, fue una de las características que más gustó al alumnado, especialmente el rol del fotógrafo. Es necesario destacar que para potenciar este rol fue necesario la elaboración de un blog (http://blogs.iesealarcos.es/ campeonato/) en el que se mostraban las fotografías que ellos mismos capturaban, además de otras cuestiones de interés (clasificaciones, vídeos educativos, acceso a la WebQuest, etc.). Sin el apoyo de este blog, el rol del fotógrafo hubiera sido muy débil y con poca aceptación por parte del alumnado. El resto de roles fueron bien comprendidos y desempeñados por los alumnos a pesar de no estar acostumbrados a este tipo de trabajo.

Los materiales autoconstruidos fue otro punto reseñable de la intervención. Fueron un recurso muy útil para favorecer la afiliación del equipo y al mismo tiempo, generar responsabilidad personal y social. Los equipos se juntaban para la elaboración de las banderas y camisetas. Además, tenían que traer en cada sesión su camiseta autoconstruida para obtener puntos para el equipo, generando así responsabilidad. Además, la elaboración de trofeos autoconstruidos para su entrega en la festividad final a la persona o equipo que cada uno consideró fue un gesto de reconocimiento del trabajo hacia otros alumnos/equipos que gustó mucho al alumnado.

Respecto a los objetivos didácticos, los resultados obtenidos fueron muy positivos, cumpliendo con éxito los que se propusieron en la UD. El kickboxing fue muy bien aceptado por el alumnado, especialmente por la novedad del mismo. Es importante destacar que algunos de los alumnos creían que iban a practicar kickboxing en su modalidad de contacto pleno y en cierta manera, las primeras sesiones fueron un tanto confusas para ellos porque no comprendían cómos se iba a abordar este 
deporte. Poco a poco entendieron la dinámica de la intervención, destacando el desarrollo de las formas musicales, una disciplina de equipo y de trabajo rítmico-musical que fue muy bien recibida por el alumnado por las posibilidades de incluir materiales y habilidades motrices, en combinación con las técnicas de kickboxing aprendidas a lo largo de la UD. Los alumnos cambiaron totalmente la perspectiva que tenían de este deporte, al que asociaban con conductas violentas al iniciar la intervención. Los resultados mostraron que tras la experiencia, el kickboxing era visto como un deporte más, como podía ser el fútbol o el bádminton. Asimismo, la práctica de este deporte potenció destrezas técnicas que tradicionalmente no están desarrolladas en el alumnado por la ausencia de deportes similares en el ámbito de la EF. La mejora coordinativa mano-pie fue muy significativa, a lo que se le suman cuestiones como los desplazamientos, el equilibrio o los propios golpeos de este deporte. Finalmente, a nivel comportamental, el modelo híbrido propició una mejora general de conductas de responsabilidad personal y social que favorecieron la creación de un clima de aula positivo.

En referencia a las competencias, estas se han visto cumplidas de forma muy positiva. No desglosaremos la contribución de la UD a la competencia cultural y artística, social y ciudadana y autonomía e iniciativa personal por la similitud que guarda con los resultados de la experiencia en relación a los objetivos didácticos antes explicados. Sin embargo, es menester citar la contribución de esta intervención a la competencia digital puesto que en ella, el alumnado accedía diariamente al blog para ver las fotografías, clasificaciones u otros materiales que se presentaban en el mismo. Además, la utilización de la WebQuest como examen conceptual y como elemento para obtener puntos de equipo propició que el alumnado utilizara el uso de las Tecnologías de la Información y Comunicación (TIC), tan requeridas en la sociedad actual.

Finalmente, respecto a los instrumentos de evaluación, los resultados han sido nuevamente muy positivos. La utilización de la escala de valoración procedimental fue un instrumento sencillo y muy útil para valorar al alumnado en este aspecto. En referencia a la evaluación comportamental, utilizar las autoevaluaciones de los alumnos, en conjunción con la del profesor, fue un instrumento de mucho valor que ayudó a orientar a los alumnos hacia una evaluación crítica y justa (responsabilidad). No obstante, es importante destacar que una gran parte del alumnado, especialmente al inicio de la UD, no se atrevía a calificarse con la nota que realmente merecían, puntuándose generalmente por debajo, bien por miedo a lo que pudiera pensar el profesor, o los compañeros (especialmente los de su mismo equipo). En otro sentido, se dieron algunos casos de alumnos que se autoevaluaban de forma totalmente contraria, puntuándose muy alto cuando no merecían esa nota. Esto molestó mucho a aquellos alumnos que sí lo habían hecho de forma correcta. Para paliar este problema, en primer lugar, en los encuentros de grupo se debatió sobre estas cuestiones en muchas ocasiones, en aras de orientar a los alumnos hacia evaluaciones lógicas y justas. En segundo lugar, los escasos alumnos que continuaban con la misma dinámica de puntuarse muy alto sin merecerlo, el filtro del profesor fue necesario para que la calificación comportamental fuera razonada y no ocasionara enfados al resto del alumnado. Este filtro que el profesor utilizó lo expuso en los encuentros grupales para tratar de reconducir los comportamientos de los alumnos que reincidían en la misma cuestión. Para finalizar, en relación a la evaluación conceptual, la utilización de la WebQuest como instrumento fue muy enriquecedora. Obligó a los estudiantes a utilizar las TIC, y por ende, a manejar recursos visuales y textuales a través de Internet, fomentando una mejora en la competencia digital antes mencionada.

\section{Conclusiones}

La EF actual demanda planteamientos innovadores que enganchen a todo tipo de alumnado. Los modelos pedagógicos ofrecen posibilidades que los planteamientos tradicionales no permiten. La combinación o hibridación de los modelos de Educación Deportiva y Responsabilidad Personal y Social permite transformar el aula en un contexto centrado en el estudiante. Además, estos enfoques propician al alumnado de experiencias deportivas auténticas y posibilitan el desarrollo de la res- ponsabilidad personal y social a través de la actividad física. El kickboxing educativo (sin contacto) se ha mostrado como un contenido muy válido para la EF, especialmente por su carácter novedoso. Los alumnos practicaron un deporte que les produjo unos significativos beneficios a nivel motor y ayudó a que cambiaran la percepción que tenían sobre esta práctica. La disciplina de las formas musicales fue una modalidad con gran potencial educativo por su naturaleza cooperativa e innovadora. Las limitaciones principales de esta intervención fueron la inexistente exposición previa de los alumnos a los modelos y al contenido. Asimismo, el profesor tampoco tenía bagaje en lo referido al MED y al TPSR, lo que dificultó en algunas ocasiones el correcto desarrollo de la intervención. Las futuras líneas de investigación pueden centrarse en continuar hibridando estos modelos a través otro tipo de deportes para comprobar en qué grado puede afectar un contenido innovador (kickboxing) con uno tradicional (e.g. baloncesto, voleibol) en un modelo híbrido como el que aquí se ha presentado. Asimismo, es necesario continuar realizando hibridaciones de estos modelos con otros como el Aprendizaje Cooperativo o la Enseñanza Comprensiva. Otra opción muy interesante sería aplicar este contenido en estos modelos citados.

\section{Agradecimientos}

Al IES Emilio Alarcos (Gijón, Asturias) por apoyar este proyecto.

\section{Referencias}

Buchanan,A. M. (2001). Contextual challenges to teaching responsibility in a sports camp. Journal of Teaching in Physical Education, 20, 155-171.

Caballero, P.J. (2012). Diseño y evaluación de un programa de responsabilidad personal y social a través de actividad física en el medio natural en alumnado de formación profesional. (Tesis doctoral). Universidad Pablo de Olavide, Sevilla.

Caballero, P.J. (2015). Diseño, implementación y evaluación de un programa de actividades en la naturaleza para promover la responsabilidad personal y social en alumnos de formación profesional. Cuadernos de Psicología del Deporte, 15(2), 179-194.

Caballero P.J., Delgado-Noguera, M.A. y Escartí-Carbonell, A. (2013). Analysis of Teaching and Personal Social Responsibility modelbased programmmes applied in USA and Spain. Journal of Human Sport and Exercise, 8(2), 427-441.

Calderón, A., Martínez de Ojeda, D. y Martínez, I.M. (2013). Influencia de la habilidad física percibida tras una unidad didáctica basada en Educación Deportiva. Retos. Nuevas Tendencias en Educación Física, Deporte y Recreación, 24, 16-20.

Carreres, F. (2014). Efectos, en los adolescentes, de un programa de responsabilidad personal y social a través del deporte extraescolar. (Tesis doctoral). Universidad de Alicante, Alicante.

Casey, A., Dyson, B., \& Campbell,A. (2009). Action research in physical education: Focusing beyond myself through cooperative learning. Educational Action Research, 17, 407-423

Cuevas, R., García, L.M. y Contreras, O. (2015). Influencia del modelo de Educación Deportiva en las necesidades psicológicas básicas. Cuadernos de Psicología del Deporte, 15(2), 155-162.

Decreto 74/2007, de 14 de junio, por el que se regula la ordenación y establece el currículo de Educación secundaria obligatoria en el Principado de Asturias. Recuperado de https://sede.asturias.es/bopa/ disposiciones/repositorio/LEGISLACION34/66/14/ 6CE1B1FF8CF14947AFC0124183FE4737.pdf [Consultado 12/ 09/2015].

Escartí, A., Gutiérrez, M., Pascual, C., Marín, D., Martínez, C., \& Chacón, Y.(2006). Enseñando Responsabilidad Personal y Social a un grupo de adolescentes de riesgo: Un estudio observacional. Revista de Educación, 341, 373-396.

Farias, C.F., Mesquita, I.R. y Hastie, P.A. (2015). Game performance and understanding within a hybrid Sport Education season. Journal of Teaching in Physical Education, 34(3), 363-383. doi: 10.1123/ jtpe.2013-0149

Fernández-Río, J. (2006). Estructuras de trabajo cooperativas, apren- 
dizaje a través de claves y pensamiento crítico en la enseñanza de los deportes en el ámbito educativo. Actas del V Congreso Internacional deActividades Físicas Cooperativas. Coruña: Universidade da Coruña.

Fernandez-Rio, J. (2014). Another step in Models-based practice: hybridizing Cooperative Learning and Teaching for Personal and Social Responsibility. Journal of Physical Education, Recreation and Dance, 85(7), 3-5.

Fernández-Río, J. (2015). Models-based Practice Reloaded: Connecting Cooperative Learning andAdventure Education. Journal of Physical Education, Recreation \& Dance, 86(6), 5-7.

Fernández-Río, J. y Méndez-Giménez, A. (2013). La facilitación de todo el proceso de enseñanza-aprendizaje en Educación Física a través del uso de claves. Retos. Nuevas Tendencias en Educación Física, Deporte y Recreación, 24, 5-8

Fernández-Río, J. y Méndez-Giménez, A. (2016). El Aprendizaje Cooperativo: Modelo Pedagógico para Educación Física. Retos. Nuevas Tendencias en Educación Física, Deporte y Recreación, 29, 201-206.

García, L.M., Gutiérrez, D., González, S. y Valero, A. (2012). Cambios en la Empatía, la Asertividad y las Relaciones Sociales por la Aplicación del Modelo de Instrucción Educación Deportiva. Revista de Psicología del Deporte, 21(2), 321-330.

García, L.M., y Gutiérrez, D. (2015). The effects of a sport education season on empathy and assertiveness. Physical Education \& Sport Pedagogy, 20(1), 1-16. doi: 10.1080/17408989.2013.780592

Gutiérrez, D., García, L.M., Chaparro, R. y Fernández, A.J. (2014). Aplicaciones del modelo de Educación Deportiva en segundo de Educación Primaria. Percepciones del alumnado y el profesorado. Cuadernos de Psicología del Deporte, 14(2), 131-144.

Haerens, L., Kirk, D. Cardon, G. y De Bourdeaudhuij, I. (2011) Toward the Development of a Pedagogical Model for Health-Based Physical Education, Quest, 63(3), 321-338. doi: 10.1080/ 00336297.2011.10483684

Harvey, S., Kirk, D., y O’Donovan, T. M. (2014). Sport education as a pedagogical application for ethical development in physical education and youth sport. Sport, Education and Society, 19(1), 41-62. doi: 10.1080/13573322.2011.624594

Hastie, P.A. (1996). Student role involvement during a unit of sport education. Journal of Teaching in Physical Education, 16, 88-103

Hastie, P.A. y Buchanan, A.M. (2000). Teaching responsibility through sport education: Prospects of a coalition. Research Quarterly for Exercise and Sport 71(4), 25-35. doi: 10.1080/ 02701367.2000.10608877

Hastie, P. A. y Casey, A. (2014). Fidelity in Models-Based Practice Research in Sport Pedagogy: A Guide for Future Investigations. Journal of Teaching in Physical Education, 33, 422-431.

Hastie, P.A. y Sinelnikov, O.A. (2006). Russian students' participation in an perceptions of a season of Sport Education. European Physical Education Review, 12(2), 131-151. doi: 10.1177/ 1356336X06065166

Hellison, D. (Ed.) (2011). Teaching responsibility through physical activity (3er Ed.). Champaign, IL: Human Kinetics.

Jewett, A.E., Bain, L.L. y Ennis, C.D. (1995). The curriculum process in physical education. Dubuque, IA: Brown \& Benchmark.

Joyce, B. y Weil, M. (1985). Modelos de enseñanza. Madrid: Anaya.

Ley Orgánica 2/2006, de 3 de mayo, de educación. Recuperado de http:/ /www.boe.es/buscar/act.php?id=BOE-A-2006-7899 [Consultado 12/09/2015]

Mahadero, P, Calderón, A., Arias-Estero, J.L., Hastie, P.A. y Guarino, A.J. (2015). Effects of student skill level on knowledge, decision making, skill execution and game performance in a mini-volleyball Sport Education season. Journal of Teaching in Physical Education, 34(4), 626-641. doi: 10.1123/jtpe.2014-0061

Marín, D. (2011). Adaptación e implementación de un programa de intervención en la escuela a través de la educación física: el programa de responsabilidad personal y social. (Tesis doctoral). Uni- versidad de Valencia, Valencia.

Martinek, T. y Hellison, D. (1997). Service-bonded inquiry: The road less traveled. Journal of Teaching in Physical Education, 17, 107121

Menéndez, J.I. y Fernández-Rio, J. (2014a). Innovación en Educación Física: el kickboxing como contenido educativo. Apunts: Educación Física y Deportes, 11(3), 33-42. doi: 10.5672/apunts.20140983.es.(2014/3).117.03

Menéndez, J.I. y Fernández-Río, J. (2014b). Valoración de jóvenes escolares ante una experiencia de kickboxing educativo. Revista EmásF, 27, 51-63.

Meroño, L., Calderón, A. y Hastie, P.A. (2015). Efectos de una intervención basada en el modelo de Educación Deportiva sobre variables psicológicas en nadadores federados. Cuadernos de Psicología del Deporte, 15(2), 35-46.

Metzler, M.W. (2011). Instructional models for physical education ( $3^{\text {th }}$ Ed.). Scottsdale, AZ: Holcomb Hathaway.

Mosston, M. y Ashworth, S. (2002). Teaching physical education (5 $5^{\text {th }}$ Ed.). San Francisco: B. Cummings.

Pereira, J., Hastie, P.A., Araújo, R., Farias, C., Rolim, R. y Mesquita, I. (2015). A comparative study of students' track and field technical performance in SportEducation and in a direct instruction approach. Journal of Sports Science and Medicine, 14, 118-127.

Rovegno, I. (2006). Teaching and learning tactical game play at the elementary school level: the role of situated cognition. In: Wallian, N., Poggi, M-P., \& Musard, M. (Eds), Co-construire des savoirs: les metiers de l'intervention dans les APSP (pp. 115-126).Besançon: Presses universitaries de Franche-Comte.

Siedentop, D. (1994). Sport education: Quality PE through positive sportexperiences. Champaign, IL: Human Kinetics.

Siedentop, D., Hastie, P. A. y Van Der Mars, H. (2011). Complete Guide to Sport Education ( ${ }^{\mathrm{a}}$ Ed.), Champaign, IL, Human Kinetics.

Van Dalen, D. y Bennet, B. (1971). A world history of physical education: Cultural, philosophical, comparative (2nd Ed.). Englewood Cliffs, NJ: Prentice Hall.

Venero, J.P.(2007). La clase de Educación Física como motor de cambio social. Reflexionando sobre actividades en la naturaleza, curriculum oculto y valores. Nuevas Tendencias en Educación Física, Deporte y Recreación, 11, 51-53.

Vertonghen, J. y Theeboom, M. (2010). The social-psychological outcomes of martial arts practice among youth: a review. Journal of Sports Science and Medicine, 9, 528-537.

WAKO (2011). Reglamento de Formas Musicales. Recuperado de http://www.wakomexico.com/attachments/Capitulo4_Espanol.pdf [Consultado 12/09/2015].

Wright, P.M. (2001). Teaching holistic physical activity for personal and social development. Unpublished doctoral dissertation, University of Illinois at Chicago.

Wright, P.M. (2002). Violence prevention: What can coaches and sport educators do? In B.J. Lombardo, T.J. Caravella-Nadeau, K.S. Castagno, \& V.H. Mancini(Eds.), Sport in the twenty-first century: Alternatives for the new millennium (pp. 189-202). Boston: Pearson.

Wright, P.M. y Burton, S. (2008). Implementation and outcomes of a responsibility-based physical activity program integrated into an intact high school Physical Education class. Journal of Teaching in Physical Education, 27, 138-154.

Wright, P.M., Dyson, B. y Moten, T. (2012). Exploring the individualized experiences of participants in a responsibility-based youth development program. Ágora, 14(2), 248-263.

Wright, P.M., Li, W., Ding, S. y Pickering, M. (2010). Integrating a personal and social responsibility program into a Wellness course for urban high school students: assessing implementation and educational outcomes. Sport, Education \& Society, 15(3), 277-298. doi: 10.1080/13573322.2010.493309

Wright, P.M., White, K. y Gaebler-Spira, D. (2004). Exploring the Relevance of the Personal and Social Responsibility Model in Adapted Physical Activity: A Collective Case Study. Journal of Teaching in Physical Education, 23, 71-87. 